\title{
Cold-adapted RTX lipase from antarctic Pseudomonas sp. strain AMS8 : isolation, molecular modeling and heterologous expression.
}

\begin{abstract}
A new strain of psychrophilic bacteria (designated strain AMS8) from Antarctic soil was screened for extracellular lipolytic activity and further analyzed using molecular approach. Analysis of $16 \mathrm{~S}$ rDNA showed that strain AMS8 was similar to Pseudomonas sp. A lipase gene named lipAMS8 was successfully isolated from strain AMS8, cloned, sequenced and overexpressed in Escherichia coli. Sequence analysis revealed that lipAMS8 consist of 1,431 bp nucleotides that encoded a polypeptide consisting of 476 amino acids. It lacked an Nterminal signal peptide and contained a glycine- and aspartate-rich nonapeptide sequence at the C-terminus, which are known to be the characteristics of repeats-in-toxin bacterial lipases. Furthermore, the substrate binding site of lipAMS8 was identified as S207, D 255 and H313, based on homology modeling and multiple sequence alignment. Crude lipase exhibited maximum activity at $20 \mathrm{C}$ and retained almost $50 \%$ of its activity at $10 \mathrm{C}$. The molecular weight of lipAMS8 was estimated to be $50 \mathrm{kDa}$ via sodium dodecyl sulfate-polyacrylamide gel electrophoresis (SDS-PAGE). The optimal expression level was attained using the recombinant plasmid pET32b/BL21(DE3) expressed at $15 \mathrm{C}$ for $8 \mathrm{~h}$, induced by $0.1 \mathrm{mM}$ isopropyl $\beta$-D thiogalactoside (IPTG) at E. coli growth optimal density of 0.5 .
\end{abstract}

Keyword: Cold-adapted; lipAMS8; RTX repeats; Signal peptide. 